\title{
REVISÃO NOMENCLATURAL E TAXONÔMICA DE LIQUENS FOLIÍCOLAS E RESPECTIVOS FUNGOS LIQUENÍCOLAS REGISTRADOS PARA O ESTADO DE PERNAMBUCO, BRASIL, POR BATISTA E COLABORADORES
}

\author{
Robert Lücking ${ }^{\mathrm{t}}$ \\ Marcela E. da Silva Cáceres ${ }^{2}$ \\ Leonor Costa Maia $^{2}$
}

Recebido em 12/11/1998. Aceito em 07/04/1999

\begin{abstract}
RESUMO - (Revisão nomenclatural e taxonômica de liquens foliícolas e respectivos fungos liquenícolas registrados para o Estado de Pernambuco, Brasil, por Batista e colaboradores). Foram revisadas as coleções de liquens foliícolas realizadas pelo micologista Augusto Chaves Batista e seus colaboradores no Estado de Pernambuco. O estudo foi desenvolvido em três partes: (1) compilação e revisão nomenclatural dos nomes aplicados por Batista et al.; (2) revisão taxonômica dos espécimes determinados por Batista et al.; (3) identificação dos espécimes também presentes nas coleções, porém não registrados por Batista et al. As coleções estudadas compreendem 474 exsicatas com 1.130 espécimes identificados por Batista e seu grupo, nas quais esses autores aplicaram 55 nomes genéricos e 159 nomes específicos. A revisão nomenclatural aqui apresentada demonstrou que esses correspondem a 36 nomes genéricos e 101 nomes específicos válidos, mais 11 nomina dubia. No entanto, a revisão taxonômica desses 1.130 espécimes revelou que apenas 37 espécies em 16 gêneros foram corretamente identificadas, enquanto que os demais nomes representam determinações incorretas. No presente estudo, foram identificados 1.670 espécimes adicionais que não haviam sido registrados por Batista et al, Como resultado final da pesquisa, foram revisados 2.800 espécimes, os quais atualmente agrupam-se em 37 gêneros e 134 espécies de liquens foliícolas e/ou fungos liquenícolas.
\end{abstract}

Palavras-chave - liquens foliícolas, Brasil, nordeste, Chaves Batista

\begin{abstract}
Taxonomic revision of foliicolous lichens and their lichenicolous fungi as registered for Pernambuco State, Brazil, by Batista and co-workers). We revised the collections of foliicolous lichens made by the Brazilian mycologist Augusto Chaves Batista and his coworkers in Pernambuco state, Brazil. The study was divided into three parts: (1) compilation and nomenclatural revision of the names published and used by Batista et al.; (2) taxonomic revision of the specimens determined by Batista et al.; (3) identification of specimens present in the collections but not registered by Batista et al. The studied material comprises 474 collections, with 1,130 specimens identified by Batista and his group, to which these authors applied 55 generic and 159 specific names. The nomenclatural revision presented here showed that these correspond to 36 valid generic and 101 valid specific names, besides 11 nomina dubia. However, the taxonomic revision of these 1,130 specimens revealed that no more than 37 species in 16 genera were correctly identified, whereas the remaining names represent incorrect determinations. In the present study, we identified an additional 1,670 specimens not recognized before by Batista et al. As a final result of this investigation, we revised 2.800 specimens which actually belong to 37 genera and 134 species of foliicolous lichens and/or lichenicolous fungi.
\end{abstract}

Key words - foliicolous lichens, Brazil, Northeast, Chaves Batista

\footnotetext{
Institut fur Pflanzensystematik, Universität Bayreuth, D95447 Bayreuth, Alemanha

2 Departamento de Micologia, CCB, Universidade Federal de Pernambuco, Av. Prof. Moraes Rego s/nº, CEP 50670-420, Recife, Pernambuco, Brasil
} 


\section{Inrodução}

Em 1952, o liquenologista sueco Rolf Santesson publicou sua monografia mundial sobre liquens foliícolas, os quais crescem sobre folhas de plantas vasculares e têm maior diversidade nas florestas tropicais úmidas (Sérusiaux 1989; Lücking 1995). Logo depois, em 1954, o micologista Augusto Chaves Batista fundou o Instituto de Micologia, em Recife (Carneiro 1968; Singer 1969). Batista começou a estudar os liquens folíícolas do Brasil e criou um grupo de trabalho com vários colaboradores que coletaram e identificaram material de várias partes do país, especialmente da região Amazônica (Estados de Roraima, Amazonas, Rondônia, Amapá, Pará e Maranhão) e do Estado de Pernambuco, na região nordeste. Acumularam cerca de 9.000 coleções com 18.000 espécimes e publicaram em torno de 250 nomes de liquens foliícolas entre 1960 e 1975, entre os quais 38 gêneros e 68 espécies novas (Silva \& Minter 1995; Lücking et al. 1998).

Em estudo anterior, Lücking et al. (1998) revisaram os táxons de liquens foliícolas descritos por Batista et al. e verificaram que a maioria deles constitui sinônimos de gêneros e espécies previamente descritas. A grande quantidade de sinônimos se deve ao fato de Batista e seu grupo tratarem teleomorfos (indivíduos com reprodução sexuada) e anamorfos (indivíduos com reprodução assexuada) como táxons nomenclaturalmente separados. Este procedimento é permitido em fungos não liquenizados devido ao grande número de espécies cujas fases assexuadas têm importância econômica e, tradicionalmente, são identificadas com nome diferente dado à fase sexuada. Nesses casos, o nome do anamorfo é considerado artificial e não tem prioridade nomenclatural em relação ao nome do teleomorfo (ICBN Art. 59.1). No entanto, essa mesma regra exclui os fungos liquenizados (liquens), de maneira que, nesses organismos, não é possível a existência de nomes artificiais para anamorfos ou nomes diferentes para o anamorfo e o teleomorfo do mesmo táxon.

Os ambientes principais para liquens foliícolas em Pernambuco são os remanescentes da Mata Atlântica. Esse ecossistema formou originalmente faixa contínua desde o nordeste até o sudeste do Brasil, mas hoje em dia corresponde a apenas $5 \%$ da sua cobertura original (Whitmore 1990). O grau de desmatamento é ainda maior em Pernambuco, onde restam somente $2 \%$ dessa vegetação (FIDEM 1987). O grande número de liquens foliícolas registrados para esse Estado por Batista e seu grupo (Silva \& Minter 1995) indica elevada diversidade desses organismos nas áreas de Mata Atlântica. Por outro lado, os resultados da revisão realizada por Lücking et al. (1998) colocaram em dúvida a validade das respectivas identificações.

Em vista dessa situação, os objetivos do presente trabalho foram: (1) compilar lista dos nomes de liquens foliícolas registrados por Batista et al. para o Estado de Pernambuco, e realizar a revisão nomenclatural desses nomes; (2) localizar as coleções e espécimes determinados por Batista et al. e fazer revisão taxonômica desses; (3) observar espécies adicionais eventualmente presentes nas coleções e identificá-las. Os resultados deste estudo também servem como referência para comparação com levantamento atual dos liquens foliícolas de Pernambuco, que está sendo elaborado pela segunda autora deste trabalho.

\section{Material e métodos}

Foram localizadas e revisadas todas as coleções de liquens foliícolas do herbário URM registradas para Pernambuco por Batista e seus colaboradores. Como base para consulta dos nomes publicados por esses autores foram usadas as referências de Silva \& Minter (1995) e Lücking et al. (1998). A validade de cada nome foi verificada em Lücking et al. (1998) e em lista de espécies em nível mundial de Farkas \& Sipman (1997), com versão atualizada disponível na Internet (Lücking et al. 1999). Os nomes não encontrados nessas listas e os apenas usados no herbário foram identificados com base no materialtipo designado, quando acessível.

A característica das coleções de Batista e seu grupo é que cada exsicata geralmente contém várias folhas da mesma matriz, com diversos espécimes. Por esta razão, o primeiro passo do 
trabalho taxonômico foi relacionar os nomes escritos na etiqueta da exsicata com os espécimes correspondentes na coleção e reconhecer espécimes adicionalmente presentes, porém não registrados por Batista et al.

A identificação de cada espécime foi realizada mediante estudos de morfologia e anatomia. Para separar grupos maiores, foram observados o tipo do corpo de frutificação, do asco e do ficobionte. A separação das espécies foi feita observando-se o tipo de ascosporo, assim como cor, forma e tamanho dos corpos de frutificação e do talo. As observações foram realizadas com o auxílio de microscópio estereoscópico e microscópio de luz. As preparações anatômicas foram feitas em água destilada. Para a observação de alguns caracteres estruturais específicos, assim como a presença ou não de determinadas reações químicas, foram empregadas soluções de lugol a $2 \%$ e de $\mathrm{KOH}$ a $10 \%$.

As espécies foram identificadas de acordo com as chaves de Santesson (1952) e Lücking (1992; 1997) para liquens foliícolas, e Matzer (1996) para fungos liquenícolas associados a estes, além do trabalho de compilação de literatura realizado por Farkas \& Sipman (1997). Como auxílio na identificação das espécies utilizou-se também base de dados do primeiro autor sobre espécimes-tipo de todas as espécies de liquens foliícolas, incluindo descrições, preparações anatômicas e fotografias. Foram reexaminados os espécimes-tipo de espécies que haviam sido descritas por Batista e seu grupo com base no material de Pernambuco.

Por limitações de espaço, não é apresentada neste trabalho a nomenclatura detalhada das espécies identificadas durante este estudo (basiônimos, outros sinônimos, localização de espécimes-tipo). Estas informações podem ser encontradas em Farkas \& Sipman (1997) e Lücking et al. (1999). Descrições e discussões detalhadas da taxonomia de cada espécie estão em elaboração. No entanto, lista completa de todas as coleções e espécimes revisados e suas respectivas correções, está depositada no herbário URM do Departamento de Micologia da UFPE.

\section{Resultados}

Durante a revisão das coleções do herbário URM foram encontradas 474 exsicatas de liquens foliícolas provenientes do Estado de Pernambuco. A maioria das coleções foi realizada no município de Recife (174), seguido de São Lourenço da Mata (57), Paulista (41), Carpina (37), Igarassu (25), Paudalho (24) e outros oito municípios (Fig. 1). Entre o total de 13 coletores, Oswaldo Soares da Silva foi o principal, com 263 exsicatas, seguido por W. A. Cavalcante, com 106, e A. A.

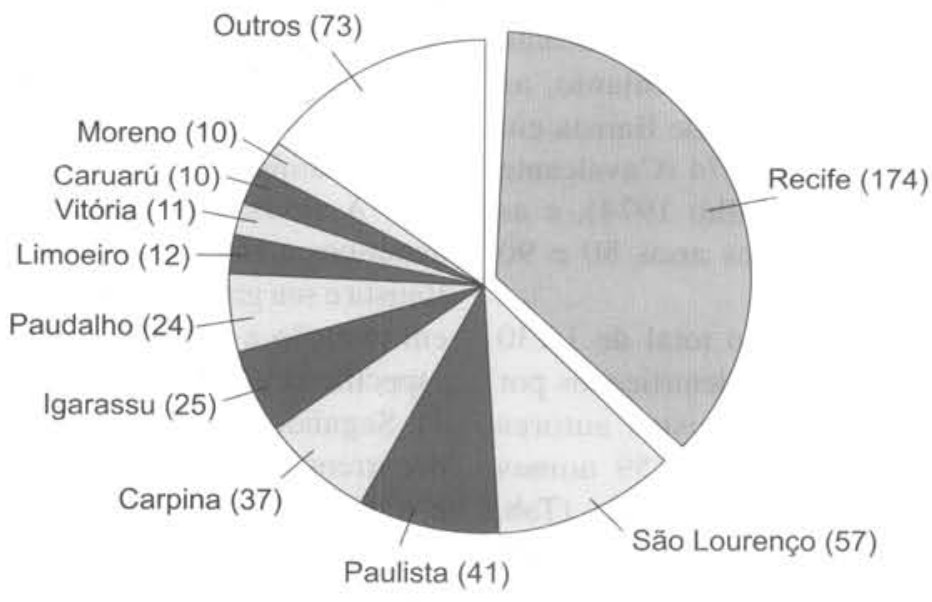

Figura 1. Número de coleções (exsicatas) de liquens foliícolas registradas por Batista e seus colaboradores em diferentes municípios do Estado de Pernambuco. 


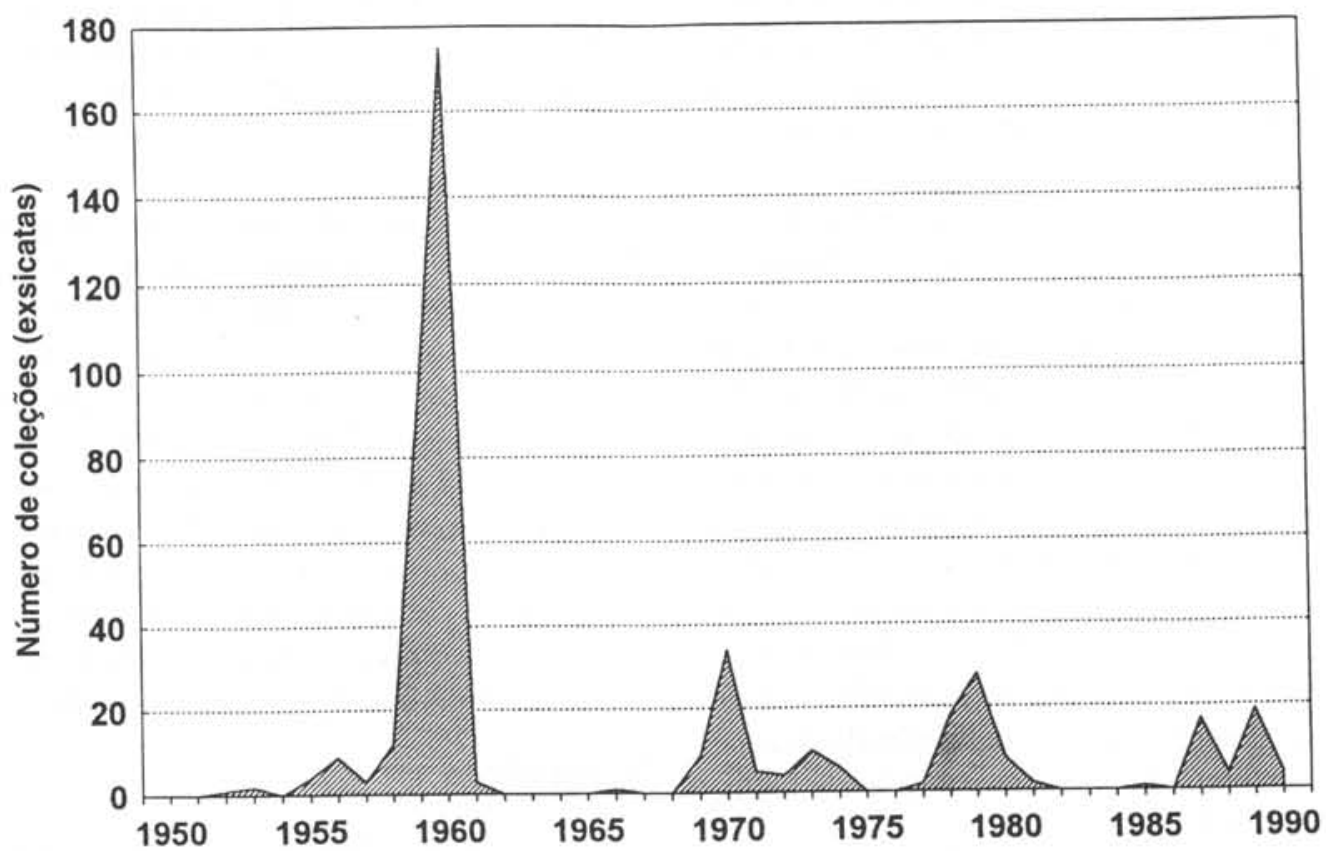

Figura 2. Distribuição temporal das coleçōes de líquens foliícolas realizadas por Batista e seus colaboradores no Estado de Pernambuco.

Silva A. Cavalcante, com 57. Outros coletores foram J. L. Bezerra, D. Poroca, G. Peres, W. Correia, o próprio A. C. Batista, R. Falcão, T. Barros, W. Gadelha, E. Luna e A. Fontes. Ordenação das coleções segundo o ano (Fig. 2) demonstra periodicidade de mais ou menos dez anos entre as coletas, com picos máximos nos anos 60 (O. Soares), 70 (principalmente D. Poroca), 80 e 90 (na sua maioria W. A. Cavalcante e A. A. Silva A. Cavalcante). No entanto, as últimas publicações, após a morte de Batista em 1967, foram feitas em 1972 e 1974 (Cavalcante et al. 1972a; 1972b; Xavier Filho 1974), e as identificações das coleções dos anos 80 e 90 nunca foram publicadas.

As 474 coleções contém o total de 1.130 espécimes de liquens foliícolas identificados por Batista e seu grupo, aos quais estes autores aplicaram 55 nomes genéricos e 159 nomes específicos, incluindo algumas variedades (Tab. 1). Das 159 espécies, 83 já foram mencionados para Pernambuco na compilação de Silva \& Minter (1995), enquanto 18 nunca foram publicadas. Os nomes aplicados por Batista et al. podem ser divididos em quatro categorias: (1) nomes baseados na monografia de Santesson (1952); (2) novos gêneros e espécies (total de 21) validamente publicados por Batista e seu grupo (Lücking et al. 1998); (3) novos táxons (total de 20) invalidamente publicados por Batista e seu grupo (Lücking et al. 1998); (4) novos táxons não publicados (total de 13), somente existentes como nomes de herbário. Dos novos táxons descritos por Batista e seus colaboradores, ou planejados para serem descritos, 54 têm sua localidade-tipo em Pernambuco.

A revisão nomenclatural dos 55 nomes genéricos e 159 nomes específicos aplicadas por Batista e seu grupo (incluindo variedades) resultou em redução a 36 nomes genéricos e 101 nomes específicos válidos, mais 11 nomina dubia (Tab. 1). Segundo Lücking et al. (1998), os sinônimos decorrentes do tratamento separado de anamorfos e teleomorfos dos mesmos táxons incluem os nomes genéricos Acleistomyces (= Sporopodium), Actinoteichus (= Asterothyrium), Crocicreomyces e Pyriomyces (= Byssoloma), Cyrta (= Calopadia), Phallomyces e Sporocybomyces (= 
Tabela 1. Nomes de gêneros e espécies de liquens foliícolas registrados por Batista e colaboradores para o Estado de Pernambuco (Silva \& Minter 1995; Lücking et al. 1998 e esta publicação) e seus nomes válidos segundo Lücking et al. (1998 e esta publicação). Nomes não incluídos na compilação de Silva \& Minter (1995) são marcados com um asterisco $(*)$. Nomes intentados como novos, mas somente encontrados no herbário, são indicados entre colchetes e aspas [" "]. A palavra "tipo" (ou "tipo designado" em casos de táxons invalidamente publicados e nomes usados no herbário) indica que o táxon respectivo tem sua localidade-tipo em Pernambuco.

Aciesia Bat. $=$ nom. dub.

Aciesia xylopiae Bat. \& J.L. Bez. (tipo) $=$ nom. dub.

Acleistomyces Bat. = Sporopodium Mont.

Acleistomyces rionegrensis Bat. et al. = Sporopodium leprieurii Mont.

Acleistomyces zollerniae Bat. \& J.A. Lima (tipo) = Sporopodium xantholeucum (Müll. Arg.) Zahlbr.

Actinoteichus Cavalc. \& Poroca $=$ Asterothyrium Müll. Arg.

*Actinoteichus aspidospermatis Peres = Asterothyrium aspidospermatis (Peres) Lücking \& Sérus.

Actinoteichus maranhensis Cavalc. \& Poroca = Asterothyrium umbilicatum (Müll. Arg.) Müll. Arg.

Actinoteichus pernambucensis Cavalc. (tipo) = Asterothyrium pernambucense (Cavalc.) Lücking \& Sérus.

*Ainsworthiomyces Bat. \& J. L. Bez. nom. inval. = nom. dub.

*Ainsworthiomyces sterculiae Bat. \& J. L. Bez. nom. inval. $=$ nom. dub.

Arthonia Ach.

*Arthonia accolens Stirt.

Arthonia anisolocularis L. Xavier (tipo) = Arthonia cyanea Müll. Arg.

*Arthonia epidendri (Rehm) R. Sant.

*Arthonia lividula Vain.

*Arthonia trilocularis Müll. Arg.

Asbolisiomyces Bat. \& H. Maia $=$ nom. dub.

Asbolisiomyces ingae Bat. \& H. Maia (tipo) $=$ nom. dub.

Aulaxina Fée

Aulaxina minuta R. Sant.

[Aulaxina "ocoteae" nom. herb. (tipo designado)] = Graphis sp.

*Aulaxina quadrangula (Stirt.) R. Sant.

Aulaxina uniseptata R. Sant.

["Aulaxinomyces" nom. herb.] = Aulaxina Fée

["Aulaxinomyces quadrangularis" comb. herb.] = Aulaxina quadrangula (Stirt.) R. Sant.

Bacidia De Not.

*Bacidia apiahica (Müll. Arg.) R. Sant. = Bacidina apiahica (Müll. Arg.) Vezda

["Bacidia" bilimbioides comb. herb.] = Tapellaria bilimbioides R. Sant.

*Bacidia gabrielis (Müll. Arg.) Zahlbr. = Loflammia gabrielis (Müll. Arg.) Vezda

[Bacidia "ingae" nom herb. (tipo designado)] = Psorotheciopsis albomaculans (Rehm) R. Sant.

* Bacidia psychotriae (Müll. Arg.) Zahlbr.

* Bacidia rhapidophylli $($ Rehm $)$ Zahlbr. = Fellhanera rhapidophylli $($ Rehm $)$ Vezda

Bacidia sublecanorina (Nyl.) Zahlbr. = Fellhanera sublecanorina (Nyl.) Vezda

*Byrsomyces Cavalc. $=$ Microtheliopsis Müll. Arg.

*Byrsomyces olivaceus Cavalc. $=$ Microtheliopsis uleana Müll. Arg.

Byssoloma Trevis.

Byssoloma chlorinum (Vain.) Zahlbr.

* Byssoloma polychromum (Müll. Arg.) Zahlbr.

*Byssoloma rotuliforme (Müll. Arg.) R. Sant. = Byssoloma subdiscordans (Nyl.) P. James

Byssoloma tricholomum (Mont.) Zahlbr.

Calenia Müll. Arg.

Calenia aggregata $\mathrm{R}$. Sant.

*Calenia depressa Müll. Arg.

*Calenia graphidea Vain.

[Calenia graphidea var. "microspora" nom. herb. (tipo designado)] = Mazosia dispersa (Hedr.) R. Sant.

*Calenia laevigata Müll. Arg. = Caleniopsis laevigata (Müll. Arg.) Vezda \& Poelt

Catenata Bat. nom. inval. $=$ Strigula Fr.

Catenata antillarum $($ Fée) Bat. comb. inval. $=$ Strigula antillarum (Fée) Müll. Arg.

Chaetomonodorus Bat. \& H. Maia = Microtheliopsis Müll. Arg.

Chaetomonodorus brosimi Bat. \& H. Maia (tipo) = Microtheliopsis uleana Müll. Arg.

Chroodiscus (Müll. Arg.) Müll. Arg.

Chroodiscus coccineus (Leight.) Müll. Arg. 
Tabela 1. (continuação)

Coenogonium Ehrenb.

* Coenogonium moniliforme Tuck.

*Crocicreomyces Bat. \& Peres = Byssoloma Trevis.

* Crocicreomyces guttiferae Bat. \& Peres = Byssoloma guttiferae (Bat. \& Peres) Lücking \& Sérus.

Cyrta Bat. \& H. Maia = Calopadia Vezda

Cyrta licaniae Bat. \& H. Maia (tipo) = Calopadia subcoerulescens (Zahlbr.) Vezda

*Didymopyrostroma Bat. \& Cavalc. nom. inval. = Lichenopeltella Höhn.

*Didymopyrostroma xylopiae Bat. \& Cavalc. nom. inval. $=$ Lichenopeltella epiphylla $\mathrm{R}$. Sant.

Echinoplaca Fée

*Echinoplaca argentea (Mont.) R. Sant. = Gyalideopsis argentea (Mont.) Kalb \& Vezda

Echinoplaca epiphylla (Fée) Fée

*Echinoplaca heterella (Stirt.) R. Sant. = Tricharia heterella $($ Stirt.) Lücking

*Enterographa Fée

[Enterographa "pernambucensis" nom. herb. (tipo designado)] = Opegrapha filicina Mont.

*Enterographa pernambucensis var. psychotriae Bat. \& Maia nom. inval. (tipo designado) = Opegrapha epiporina Matzer

["Enterographopsis" nom. herb.] = Arthonia Ach.

["Enterographopsis flacourtiae" nom. herb. (tipo designado)] = Arthonia intermedia Matzer

Gyalectidium Müll. Arg.

*Gyalectidium aspidotum (Vain.) R. Sant. = Bullatina aspidota (Vain.) Vezda \& Poelt

Gyalectidium filicinum Müll. Arg.

["Lopadiopsis" nom. herb.] = Calopadia Vezda

["Lopadiopsis lichenicola" nom. herb. (tipo designado)] = Calopadia puiggarii (Müll. Arg.) Vezda

Lopadium Körb.

Lopadium applanatum H. Maia nom. inval. (tipo designado) = Calopadia subcoerulescens (Zahlbr.) Vezda

Lopadium didymopanacis Bat. \& Peres nom, inval. (tipo designado) = Sporopodium phyllocharis (Mont.) Massal.

Lopadium epiphyllum Müll. Arg. = Tapellaria epiphylla (Müll. Arg.) R. Sant.

Lopadium flammeum Müll. Arg. = Loflammia epiphylla (Fée) Lücking \& Vezda

Lopadium fuscum Müll. Arg. = Calopadia fusca (Müll. Arg.) Vezda

* Lopadium gilvum Müll. Arg. = Logilvia gilva (Müll. Arg.) Vezda

Lopadium paudalhense Bat. \& Peres nom. inval. (tipo designado) = Tapellaria nana (Fée) R. Sant.

Lyromma Bat. \& H. Maia

Lyromma nectandrae Bat. \& H. Maia (tipo)

* Lyrommotheca Bat., Pavlich \& J. L. Bez. nom. inval. = Lyromma Bat. \& H. Maia

* Lyrommotheca leguminosarum Bat. et al. nom. inval. = Lyromma nectandrae Bat. \& H. Maia

Mazosia Massal.

Mazosia dispersa (Hedr.) R. Sant.

Mazosia melanophthalma (Müll. Arg.) R. Sant.

Mazosia paupercula var. macrospora Bat. \& H. Maia (tipo) = Mazosia praemorsa (Stirt.) R. Sant.

Mazosia phyllosema (Nyl.) Zahlbr.

Mazosia praemorsa (Stirt.) R. Sant.

Mazosia praemorsa var. macrocarpa nom. inval. (tipo designado) = Mazosia longispora Lücking \& Matzer

Mazosia rotula (Mont.) Massal.

* Mazosia tumidula (Stirt.) Müll. Arg.

*Microtheliopsis Müll. Arg.

[Microtheliopsis "licaniae" nom. herb.] = Microtheliopsis uleana Müll. Arg.

*Microxyphiomyces Bat., Valle \& Peres = Tricharia Fée

*Microxyphiomyces capitulatus Bat. \& J.L. Bez. = Tricharia vainioi R. Sant.

*Microxyphiomyces manaosensis Bat., Valle \& Peres = Tricharia vainioi $\mathrm{R}$. Sant.

*Microxyphiomyces minutus Bat. \& Cavalc. = Aulaxina minuta $\mathrm{R}$. Sant.

Monodorus Bat. nom. inval. $=$ Microtheliopsis Müll. Arg.

Monodorus hendersonianus Bat. \& J. L. Bez. nom. inval. (tipo designado) = Microtheliopsis uleana Müll. Arg.

Mysia Bat. nom. inval. $=$ nom. dub.

Mysia combreti Bat. nom. inval. (tipo designado) = nom. dub.

Mysia microspora Bat. \& Cavalc. nom. inval. $=$ nom. dub.

Opegrapha Ach.

Opegrapha filicina Mont. 
Tabela 1. (continuação)

* Opegrapha puiggarii Müll. Arg.

*Phallomyces Bat. \& Valle = Echinoplaca Fée

*Phallomyces palmae Bat. \& Valle = Echinoplaca $\mathrm{sp}$.

Phragmopeltheca L. Xavier = Porina Müll. Arg.

Phragmopeltheca caseariae L. Xavier (tipo) = Porina rubentior (Stirt.) Müll. Arg.

[Phragmopeltheca "caseariicola" Bat. \& Costa in Silva \& Minter $(1995)=$ Phragmopeltheca caesariae L. Xavier]

Phragmopeltheca cupaniae L. Xavier (tipo) = Porina rubentior (Stirt.) Müll. Arg.

Phragmopeltheca cupaniae var. caruarensis L. Xavier (tipo) = Porina ?rubentior (Stirt.) Müll. Arg.

Phragmopeltheca cupaniae var. minor L. Xavier (tipo) = Porina ?rubentior (Stirt.) Müll. Arg.

Phragmopeltheca psidii L. Xavier (tipo) = Porina ?rufula (Kremp.) Vain.

Phragmopeltheca psychotriae L. Xavier (tipo) = Porina ?rubentior (Stirt.) Müll. Arg.

Phragmopeltheca pulcherrima var. octospora L. Xavier = Porina ?rubentior (Stirt.) Müll. Arg.

Phyllophiale R. Sant.

Phyllophiale alba R. Sant.

Porina Müll. Arg.

*Porina albicera (Kremp.) Van OvereemDe Haas

Porina applanata Vain.

Porina conica R. Sant.

Porina cupreola (Müll. Arg.) F. Schill.

Porina cupreola var. ciliata Bat. \&. Taltasse nom. inval. (tipo designado) = Porina epiphylla (Fée) Fée

Porina epiphylla (Fée) Fée

* Porina imitatrix Müll. Arg.

Porina kamerunensis F. Schill.

* Porina leptosperma Müll. Arg.

*Porina limbulata (Kremp.) Vain.

[Porina lucida var. "ciliata" nom. herb. (tipo designado)] = Porina subepiphylla Lücking \& Vezda

*Porina nitidula Müll. Arg.

[Porina pallescens var. "macrocarpa" nom. herb. (tipo designado)] = Porina nitidula Müll. Arg.

* Porina rubentior (Stirt.) Müll. Arg.

Porina rufula (Kremp.) Vain.

*Porina thaxteri R. Sant.

Porina verruculosa Müll. Arg.

* Psathyromyces Bat. \& Peres = Tricharia Fée

*Psathyromyces minutus Bat. \& J. L. Bez. nom. inval. $=$ nom. dub.

Psorotheciopsis Rehm

*Psorotheciopsis albomaculans (Rehm) R. Sant.

Psorotheciopsis paudalhensis Bat. \& Peres nom. inval. (tipo designado) = Psorotheciopsis premneella (Müll. Arg.) R. Sant. Psorotheciopsis premneella (Müll. Arg.) R. Sant.

Pycnociliospora Bat. = Strigula $\mathrm{Fr}$.

Pycnociliospora belluciae Bat. \& J.A. Lima = Strigula antillarum (Fée) Müll. Arg.

Pycnociliospora caesalpiniifolii Bat. \& J.A. Lima (tipo) = Strigula nitidula Mont.

Pycnociliospora crescentiae Bat. \& Taltasse (tipo) = Strigula smaragdula Fr.

Pycnociliospora crescentiae var. microcarpa Bat. \& Taltasse (tipo) = Strigula smaragdula Fr.

* Pyrenotrichum Mont. = Calopadia Vezda

*Pyrenotrichum splitgerberi Mont. = Calopadia $\mathrm{sp}$.

Pyriomyces Bat. \& H. Maia = Byssoloma Trevis.

Pyriomyces protii Bat. \& H. Maia (tipo) = Byssoloma subdiscordans (Nyl.) P. James

Raciborskiella $\mathrm{Höhn.} \mathrm{=} \mathrm{Strigula} \mathrm{Fr.}$

[Raciborskiella "couepiae" nom. herb. (tipo designado)] = Strigula nitidula Mont.

[Raciborskiella "ingae" nom. herb. (tipo designado)] = Strigula subtilissima (Fée) Müll. Arg.

[Raciborskiella "lafoensiae" nom. herb. (tipo designado)] = Strigula microspora Lücking

[Raciborskiella "lafoensiae var, microcarpa" nom. herb.] = nom. dub.

Raciborskiella prasina (Müll. Arg.) R. Sant. = Strigula prasina Müll. Arg.

[Raciborskiella "siparunae" nom. herb. (tipo designado)] = Strigula microspora Lücking

Raciborskiella zollerniae Bat. \& J. A. Lima nom. inval. (tipo designado)] = Strigula microspora Lücking

Ruthia Bat. nom. inval. $=$ nonlichenized fungus 
Tabela 1. (continuação)

R. perseae Bat. nom. inval. [tipo designado] = nonlichenized fungus

* Santessonia Bat. nom. inval. non Hale \& Vobis = Arthonia Ach.

[Santessonia "elegans" nom. herb.] = nom. dub.

*Santessonia trilocularis (Müll. Arg.) Bat. comb. inval. = Arthonia trilocularis Müll. Arg.

*Scutomyces J.L. Bez. \& Cavalc. = Microtheliopsis Müll. Arg.

* Scutomyces concentricus J.L. Bez. \& Cavalc. = Microtheliopsis uleana Müll. Arg.

* Septoriomyces Cavalc. \& A. A. Silva = Phyllobathelium Müll. Arg.

*Septoriomyces leguminosae Cavalc. \& A. A. Silva = Phyllobathelium leguminosae (Cavalc. \& A.A. Silva) Lücking \& Sérus.

Setomyces Bat. \& Peres nom. inval. = Tricharia Fée

Setomyces belluciae Bat. \& Peres nom. inval. = Tricharia urceolata (Müll. Arg.) R. Sant.

Setomyces crescentiae Bat. \& Taltasse nom. inval. (tipo designado) = Tricharia vainioi $\mathrm{R}$. Sant.

Setomyces giganteae Bat. \& J. L. Bez. nom. inval. (tipo designado) = Tricharia amazonum Vain.

Setomyces minutus Bat. \&. H. Maia nom. inval. (tipo designado) = Tricharia ?vainioi R. Sant.

Setomyces orchideae Bat. \& Peres nom. inval. = Tricharia $?$ vainioi $\mathrm{R}$. Sant.

Spinomyces Bat. \& Peres nom. inval. = Echinoplaca Fée

Spinomyces genipae Bat. \& Peres nom. inval. (tipo designado) = Echinoplaca epiphylla Fée

* Spinomyces giganteae Bat. \& M. M. Herrera nom. inval. $=$ nom. dub.

*Spinomyces ocoteae Bat. \& H. Maia nom. inval. = nom. dub.

Sporocybomyces $\mathrm{H}$. Maia = Echinoplaca Fée .

Sporocybomyces pulcher H. Maia (tipo) = Echinoplaca leucotrichoides (Müll. Arg.) R. Sant.

Stephosia Bat. \& H. Maia = Phyllophiale R. Sant.

Stephosia protii Bat. \& H. Maia (tipo) = Phyllophiale alba $\mathrm{R}$. Sant.

Strigula Fr.

Strigula antillarum (Fée) R. Sant. comb. inval. = Strigula antillarum (Fée) Müll. Arg.

* Strigula complanata (Fée) Mont. = Strigula orbicularis $\mathrm{Fr}$.

Strigula concreta $($ Fée) R. Sant. = Strigula concreta $($ Fée) R. Sant.

Strigula elegans (Fée) Müll. Arg. = Strigula smaragdula Fr.

Strigula elegans var. antillarum (Fée) R. Sant. = Strigula antillarum (Fée) Müll. Arg.

Strigula hymenaeicola Bat. \& J. L. Bez. nom. inval. (tipo designado) = Strigula microspora Lücking

Strigula maculata (Cooke \& Massee) R. Sant.

Strigula melanobapha (Kremp.) R. Sant.

[Strigula melanobapha var. "minor" nom. herb. (tipo designado)] = Strigula subtilissima (Fée) Müll. Arg.

Strigula nemathora Mont.

* Strigula nitidula Mont.

[Strigula "orchyzospora" R. Sant. = Strigula schizospora R. Sant.]

Strigula schizospora R. Sant.

Strigula subtilissima (Fée) Müll. Arg.

* Strigula xylopiae Bat. \& Cavalc. = Strigula schizospora $\mathrm{R}$. Sant.

*Tauromyces Cavalc. \& A. A. Silva = Gyalectidium Müll. Arg.

*Tauronyces catenulatus Cavalc. \& A. A. Silva = Gyalectidium catenulatum (Cavalc. \& A. A. Silva) Ferraro et al. comb. ined.

*Tegaster Bat. nom. inval. $=$ nom. dub.

Tegaster protiicola Bat. \& H. Maia nom. inval. (tipo designado) nom. dub.

Tegoa Bat. \& Peres nom. inval. = Asterothyrium Müll. Arg.

*Tegoa eugeniae Bat. \& H. Maia nom. inval. (tipo designado) = Asterothyrium sp. [picnidios]

* Tegoa mappiae Bat. \& Peres nom. inval. (tipo designado) = Tapellariopsis octomera Lücking

*Tegoa parenchymatica Bat. \& Cavalc. nom. inval. = Anisomeridium foliicola $\mathrm{R}$. Sant. \& Tibell

Tegoa tabebuia Bat. \& Peres nom. inval. (tipo designado) = Asterothyrium sp. [picnidios]

*Tricharia Fée

*Tricharia carnea (Müll. Arg.) R. Sant.

*Tricharia farinosa $\mathrm{R}$. Sant.

Trichothelium Müll. Arg.

Trichothelium annulatum (Karst.) R. Sant.

*Trichothelium epiphyllum Müll. Arg.

Trichothelium epiphyllum var. "ciliatum" nom. herb. (tipo designado) = Trichothelium epiphyllum Müll. Arg. 
Echinoplaca), Psathyromyces (= Tricharia), Pycnociliospora (= Strigula), Septoriomyces (= Phyllobathelium) e Tauromyces (= Gyalectidium).

Alguns sinônimos resultam da identificação incorreta de fungos liquenícolas. O nome Didymopyrostroma, dado a espécimes com picnídios considerados como sendo de líquen, representa, na verdade, fungo liquenícola crescendo sobre talos de Porina, conhecido como Lichenopeltella (Matzer 1996; Lücking et al. 1998). Da mesma forma, Enterographopsis, nome de herbário, corresponde a Arthonia intermedia, espécie comum de fungo liquenícola também em talos de Porina. Um caso particular foi encontrado numa coleção de Calopadia puiggarii apresentando apotécios e campilídios. Estes órgãos representam, respectivamente, a reprodução sexuada e assexuada do mesmo líquen (Sérusiaux 1986; Vezda 1986). No entanto, Santesson (1952) considerou os campilídios como parte de fungo liquenícola sobre o talo com apotécios de Lopadium puiggarii (= Calopadia puiggarii). Batista e seu grupo, ao contrário, identificaram o talo com campilídios como uma espécie de liquen, e consideraram os apotécios como sendo de fungo liquenícola, denominandoos de Lopadiopsis lichenicola nom. herb.

Os nomes Byrsomyces, Chaetomonodorus e Scutomyces baseiam-se em teleomorfos (Lücking et al. 1998); no entanto, todos correspondem ao gênero Microtheliopsis. Outro nome para este mesmo gênero, Monodorus nom. inval., foi encontrado durante o presente trabalho (Tab. 1). Esta sinonímia foi estabelecida pelo fato de que a etiqueta do tipo designado continha originalmente o nome de Microtheliopsis uleana que depois foi visivelmente rasurado e substituído pelo novo nome Monodorus hendersonianus. Além disso, todas as coleções sob este nome contém espécimes de Microtheliopsis uleana. Em alguns casos, desenhos originais feitos por Batista e seus colaboradores incluídos nas coleções originais (Fig. 3) auxiliaram a identificar nomes ainda não revisados por Lücking et al. (1998). Por exemplo, a secção de apotécio de Bacidia ingae nom. herb. corresponde a Psorotheciopsis albomaculans, presente na coleção-tipo. Os ascosporos de
Calenia graphidea var. microcarpa nom. herb., descritos e desenhados nas anotações da exsicata, são característicos de Mazosia dispersa, espécie abundante na coleção-tipo. O novo táxon Ruthia perseae, anteriormente registrado como nomen dub. por Lücking et al. (1998), é fungo não liquenizado.

A revisão taxonômica dos 1.130 espécimes revelou que apenas 37 espécies em 16 gêneros foram identificados corretamente por Batista e seus colaboradores (Tab. 2), enquanto 122 registros específicos representam determinações incorretas. Os seguintes nomes exemplificam algumas identificações incorretas: Psorotheciopsis patellarioides como Aulaxina uniseptata (ambos têm apotécios com margem negra e ascosporos uniseptados, mas distinguem-se pelo tipo de talo e paráfises), Bacidia brasiliensis como Byssoloma polychromum (ambos têm talo finamente verrucoso e apotécios castanhos, mas diferem no tipo de excípulo e ascosporos), Byssoloma subdiscordans como Tapellaria bilimbioides (ambos têm talo branco disperso e apotécios negros com ascosporos 3-septados, mas possuem excípulos diferentes).

Além disso, as coleções estudadas contêm grande número de espécimes presentes nas folhas que não foram registrados anteriormente por Batista et al. Encontrou-se 1.670 espécimes adicionais, o que resulta no total de 2.800 espécimes revisados, pertencentes a 37 gêneros e 134 espécies de liquens foliícolas e/ou fungos liquenícolas a estes associados.

\section{Discussão}

A revisão nomenclatural e taxonômica dos nomes de liquens foliícolas registrados por Batista e seus colaboradores para o Estado de Pernambuco revelou alto grau de sinonímia e determinações incorretas, sendo apenas $25 \%$ dos táxons corretamente identificados. Como mencionado anteriormente, isto pode ser explicado em parte pelo fato de Batista ter sido micologista e, portanto, aplicar as mesmas regras nomenclaturais tanto para fungos não liquenizados como para liquens, usando assim nomenclatura dualista para anamorfos e teleomorfos (Lücking et al. 1998). A 
identificação incorreta de espécies deve-se também ao intercâmbio científico limitado com outros liquenologistas contemporâneos, como Rolf Santesson. Muitas espécies de liquens foliícolas, como as do gênero Porina (Lücking 1996; Lücking \& Vezda 1998), pertencem a grupos difíceis de identificação, cuja taxonomia não pode ser feita sem consulta a especialista ou exame comparativo com material de outras regiões e/ou espécimes-tipo. Em alguns casos, não foram criticamente observadas características indispensáveis para identificação correta, como a superficie do talo (no gênero Mazosia), a estrutura do excípulo do corpo de frutificação (no gênero Byssoloma), ou os ascosporos (no gênero Bacidia).

No entanto, o trabalho de Batista e seu grupo, principalmente o grande número de coleções

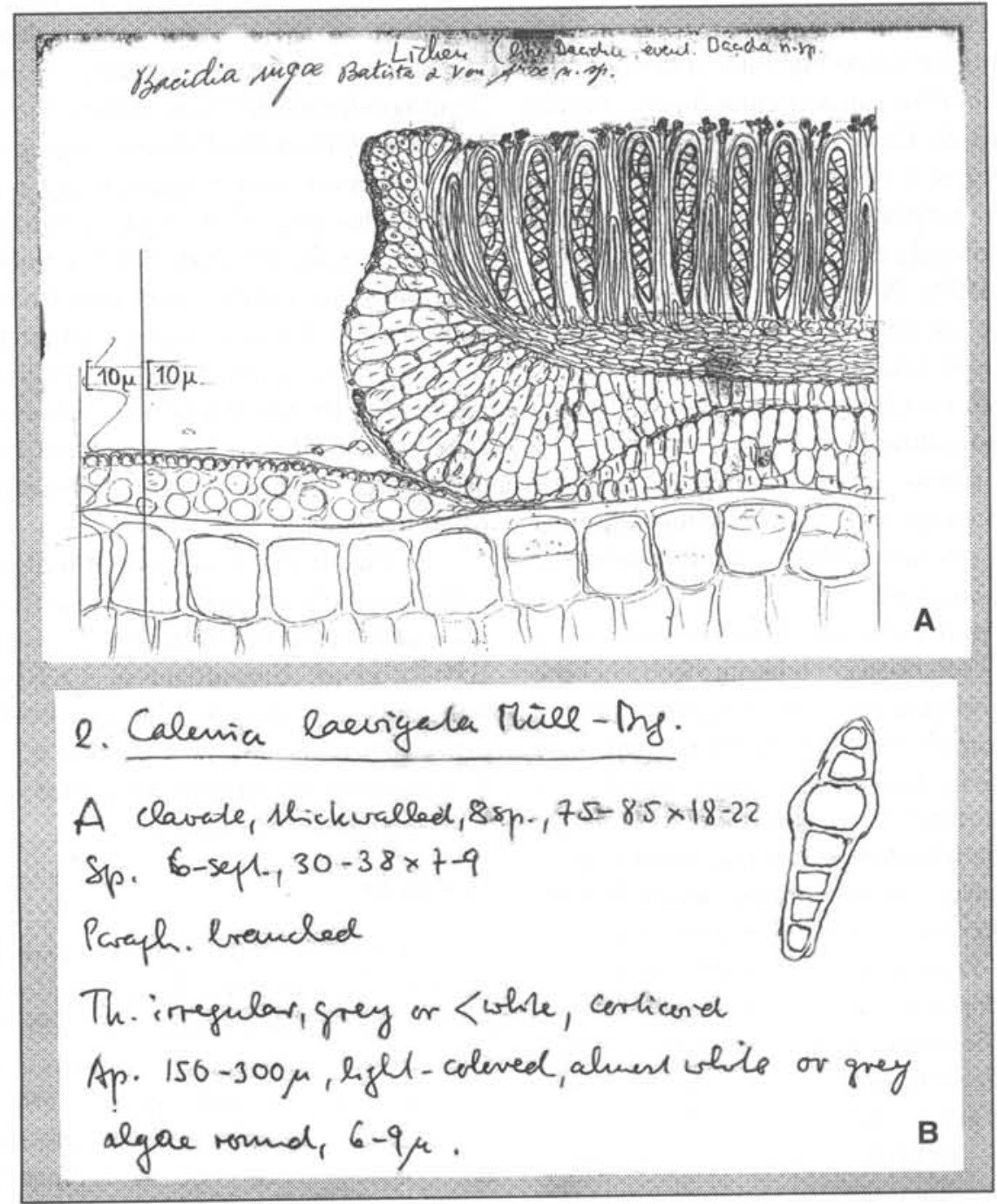

Figura 3. Cópia de desenhos originais de liquens foliícolas depositados com as coleções, feitos por J. von Arx, na época um dos cientistas visitantes no grupo de Batista. (A) Secção transversal do apotécio de Bacidia ingae nom. herb. (= Linhartia albomaculans; exs. no. 14254); o septo único e espesso dos ascosporos foi confundido com dois septos finos, assim explicando a identificação errônea como Bacidia. (B) Anotações e esboço de um ascosporo de Calenia laevigata (= Mazosia dispersa; exs. no. 14225); o esboço do ascosporo corresponde exatamente ao tipo de ascosporos encontrado no gênero Mazosia. 
Tabela 2. Gêneros e espécies de fungos liquenizados foliícolas presentes nas coleçōes de Batista e colaboradores e provenientes do Estado de Pernambuco. Táxons corretamente identificados por Batista et al. são marcados com um asterisco (*).

Actinoplaca strigulacea Müll. Arg.

Anisomeridium foliicola R. Sant. \& Tibell

*Arthonia accolens Stirt.

Arthonia aciniformis Stirt.

Arthonia atropunctata Vain. [fungo liquenícola em Echinoplaca sp.]

Arthonia cyanea Müll. Arg.

Arthonia intermedia Matzer [fungo liquenícola em Porina spp.]

Arthonia lecythidicola (Bat. \& J. L. Bez.) Lücking \& Sérus.

Arthonia leptosperma (Müll. Arg.) R. Sant.

Arthonia mira R. Sant.

Arthonia palmulacea (Müll. Arg.) R. Sant.

Arthonia pseudopegraphina Matzer [fungo liquenícola em Mazosia rubropunctata]

*Arthonia trilocularis Müll. Arg. [como Santessonia trilocularis comb. herb.]

Asterothyrium microsporum R. Sant.

Asterothyrium monosporum Müll. Arg.

Asterothyrium pittieri Müll. Arg.

Asterothyrium rotuliforme (Müll. Arg.) Sérus.

Asterothyrium tetrasporum Lücking

Aulaxina microphana (Vain.) R. Sant.

Aulaxina minuta $\mathrm{R}$. Sant.

Aulaxina opegraphina Fée

*Aulaxina quadrangula (Stirt.) R. Sant.

Bacidia brasiliensis (Müll. Arg.) Zahlbr.

Bapalmuia nigrescens (Müll. Arg.) Cáceres \& Lücking

* Bullatina aspidota (Vain.) Vezda \& Poelt

Byssolecania fumosonigricans (Müll. Arg.) R. Sant.

Byssolecania hymenocarpa (Vain.) Kalb, Vezda \& Lücking

* Byssoloma chlorinum (Vain.) Zahlbr.

Byssoloma leucoblepharum (Nyl.) Vain.

* Byssoloma subdiscordans (Nyl.) P. James [como Byssoloma rotuliforme (Müll. Arg.) R. Sant.]

* Byssoloma tricholomum (Mont.) Zahlbr.

Calenia bullatinoides Lücking ined.

Calenia conspersa (Stirt.) R. Sant.

Calenia graphidea Vain.

Calenia monospora Vezda

Calenia sp.n.

Calopadia foliicola (Fée) Vezda

* Calopadia fusca (Müll. Arg.) Vezda [como Lopadium fuscum Müll. Arg.]

Calopadia puiggarii (Müll. Arg.) Vezda

Calopadia subcoerulescens (Zahlbr.) Vezda

Caprettia amazonensis Bat. \& H. Maia

Chroodiscus australiensis Lumbsch \& Vezda

*Chroodiscus coccineus (Leight.) Müll. Arg.

Chroodiscus neotropicus Kalb \& Vezda

Dimerella epiphylla (Müll. Arg.) Malme

Dimerella flavicans Vezda \& Farkas

Echinoplaca epiphylla Fée

Echinoplaca leucotrichoides (Vain.) R. Sant.

*Echinoplaca marginata Lücking [como Echinoplaca argentea (Mont.) R. Sant.]

Echinoplaca pellicula (Müll. Arg.) R. Sant.

Echinoplaca tricharioides Kalb \& Vezda

Echinoplaca verrucifera Lücking

Eremothecella calamicola Syd.

Fellhanera bouteillei (Desm.) Vezda

*Fellhanera rhapidophylli (Rehm) Vezda [como Bacidia rhapidophylli (Rehm) R. Sant.]

Fellhanera rubida (Müll. Arg.) Zahlbr. 
Tabela 2. (continuação)

Fellhanera stanhopeae (Müll. Arg.) Lücking, Lumbsch \& Elix

Fellhanera sublecanorina (Nyl.) Vezda

Fellhanera tricharioides Lücking \& R. Sant. ined.

* Gyalectidium filicinum Müll. Arg.

Gyalideopsis cochlearifer Sérus. \& Lücking [fungo liquenícola em Echinoplaca sp.]

Gyalideopsis vulgaris (Müll. Arg.) Lücking

Hemigrapha pilocarpacearum Cáceres \& Lücking [fungo liquenícola em Byssoloma minutissimum]

Hemigrapha strigulae Matzer [fungo liquenícola em Strigula nemathora]

Keratosphaera furcatiseta Matzer [fungo liquenícola em Mazosia rotula]

Loflammia epiphylla (Fée) Lücking \& Vezda

* Lyromma nectandrae Bat. \& H. Maia

Mazosia bambusae (Vain.) R. Sant.

* Mazosia dispersa (Hedr.) R. Sant.

Mazosia longispora Lücking \& Matzer

* Mazosia melanophthalma (Müll. Arg.) R. Sant.

*Mazosia phyllosema (Nyl.) Zahlbr.

Mazosia pilosa Kalb \& Vezda

*Mazosia praemorsa (Stirt.) R. Sant.

Mazosia pseudobambusae Kalb \& Vezda

* Mazosia rotula (Mont.) Massal.

Mazosia rubropunctata $\mathrm{R}$. Sant.

Microtheliopsis uleana Müll. Arg.

Opegrapha epiporina Matzer [fungo liquenícola em Porina lucida]

* Opegrapha filicina Mont.

Opegrapha mazosiae Matzer [fungo liquenícola em Mazosia phyllosema]

Opegrapha strigulae R. Sant. ex Matzer \& R. Sant. [fungo liquenícola em Strigula smaragdula]

Phyllophiale alba R. Sant.

Porina andreana Lücking \& Vezda

Porina atrocoerulea Müll. Arg.

Porina atropunctata Lücking \& Vezda

Porina curtula Malme

Porina epilucida Aptroot \& Sipman [fungo liquenícola em Porina mirabilis]

* Porina epiphylla (Fée) Fée

Porina fulvella Müll. Arg.

Porina fusca Lücking

Porina imitatrix Müll. Arg.

Porina lucida R. Sant.

Porina mirabilis Lücking \& Vezda

*Porina nitidula Müll. Arg.

* Porina rubentior (Stirt.) Müll. Arg.

Porina rubrosphaera R. Sant.

Porina subepiphylla Lücking \& Vezda

Porina tetramera (Malme) R. Sant.

*Psorotheciopsis albomaculans (Rehm) R. Sant.

Psorotheciopsis patellarioides (Rehm) R. Sant.

* Psorotheciopsis premneella (Müll. Arg.) R. Sant.

Sporopodium leprieurii Mont.

Sporopodium phyllocharis (Mont.) Massal.

Sporopodium xantholeucum (Müll. Arg.) Zahlbr.

* Strigula antillarum (Fée) Müll. Arg. [como Strigula elegans var. antillarum (Fée) R. Sant.]

* Strigula concreta (Fée) R. Sant.

Strigula janeirensis (Müll. Arg.) Lücking

Strigula maculata (Cooke \& Massee) R. Sant.

* Strigula melanobapha (Kremp.) R. Sant.

Strigula microspora Lücking

* Strigula nemathora Mont.

* Strigula nitidula Mont. 
Tabela 2. (continuação)

*Strigula orbicularis Fr. [como Strigula complanata (Fée) Mont.]

Strigula platypoda (Müll. Arg.) R. C. Harris

* Strigula schizospora R. Sant.

* Strigula smaragdula Fr. [como Strigula elegans (Fée) Müll. Arg.]

Strigula subelegans Vain.

* Strigula subtilissima (Fée) Müll. Arg.

Strigula viridis (Lücking) R. C. Harris

Tapellaria bilimbioides $\mathrm{R}$. Sant.

Tapellaria epiphylla (Müll. Arg.) R. Sant.

Tapellaria nana (Fée) R. Sant.

Tapellariopsis octomera Lücking

Tricharia amazonum Vain.

* Tricharia carnea (Müll. Arg.) R. Sant.

* Tricharia farinosa $\mathrm{R}$. Sant.

Tricharia heterella (Stirt.) Lücking

Tricharia urceolata (Müll. Arg.) R. Sant.

Tricharia vainioi $\mathrm{R}$. Sant.

*Trichothelium bipindense F. Schill. [como Trichothelium annulatum (Karst.) R. Sant.]

*Trichothelium epiphyllum Müll. Arg.

Trichothelium minus Vain.

Vouauxiella pithospora (Cavalc. \& A. A. Silva) B. Sutton [fungo liquenícola em Porina mirabilis]

realizadas em Pernambuco e em outras regiões, representa contribuição relevante para o conhecimento dos fungos e liquens do Brasil (Silva \& Minter 1995). Apesar dos problemas taxonômicos e nomenclaturais, o número de táxons registrados por Batista et al. é representativo para a diversidade real de liquens foliícolas e fungos liquenícolas em Pernambuco. Além das 134 espécies presentes nas exsicatas do herbário URM, levantamento recente realizado pela segunda autora revelou a existência de 75 espécies adicionais, resultando assim em mais de 200 táxons presentes nos remanescentes da Mata Atlântica de Pernambuco (Cáceres, em prep.). Os liquens foliícolas geralmente têm diversidade mais elevada em florestas muito úmidas, como na região Amazônica, onde podem chegar a 300 espécies (Lücking \& Kalb 1999). A diversidade destes organismos, entre outros fatores, também é afetada por perturbações antropogênicas (Lücking 1995; 1997b). Portanto, o número de liquens foliícolas registrados para a Mata Atlântica de Pernambuco pode ser considerado relativamente alto em se tratando de região com estação seca pronunciada e, atualmente, com elevado desmatamento (FIDEM 1987; Whitmore 1990).

\section{Agradecimentos}

Robert Lücking é grato à CAPES e ao DAAD pelo suporte financeiro que permitiu a sua estada no Departamento de Micologia da UFPE como Professor Visitante, período durante o qual foi realizada a presente revisão. Marcela E. S. Cáceres (Mestrado em Biologia de Fungos) está sendo financiada pela CAPES. Leonor C. Maia agradece o apoio do CNPq (Bolsa de Produtividade em Pesquisa) e da FACEPE (Auxílio à Pesquisa).

\section{Referências bibliográficas}

Carneiro, L. de S. 1968. Augusto Chaves Batista (1916-1967). Mycologia 6: 1137-1139.

Cavalcante, W. de A.; Cavalcante, A. A. S. A. \& Leal, F. de B. (1972a). Coletânea de liquens imperfeitos. Publicações do Instituto de Micologia da Universidade Federal de Pernambuco 647: 1-46.

Cavalcante, W. de A.; Bezerra, J. L. \& Leal, F. de B. (1972b). Novos ascoliquens foliícolas de Brasil. Publicações do Instituto de Micologia da Universidade Federal de Pernambuco 675: 1-17.

Farkas, E. \& Sipman, H. J. M. 1997. Checklist of foliicolous lichenized fungi. Abstracta Botanica 21(1): 173-206.

FIDEM (1987): Reservas Ecológicas: Recife. Governo do Estado de Pernambuco, Recife. 
Lücking, R. 1992. Foliicolous lichens A contribution to the knowledge of the lichen flora of Costa Rica, Central America. Nova Hedwigia 104: 1-179.

Lücking, R. 1995. Biodiversity and conservation of foliicolous lichens in Costa Rica. Mitteilungen der Eidgenössischen Forschungsanstalt für Wald, Schnee und Landschaft 70: 63-92,

Lücking, R. 1996. Taxonomic studies in foliicolous species of the genus Porina. 1. The Porina rufula aggregate. Botanica Acta 109: 248-260.

Lücking, R. 1997a. Additions and corrections to the knowledge of the foliicolous lichen flora of Costa Rica. The family Gomphillaceae. Bibliotheca Lichenologica 65: 1-109.

Lücking, R. 1997b. The use of foliicolous lichens as bioindicators in the tropics, with special reference to the microclimate. Abstracta Botanica 21(1): 99-116.

Lücking, R. \& Kalb, K. 1999. Foliikole Flechten aus Brasilien (vornehmlich Amazonien), inklusive einer Checkliste und Bemerkungen zu Coenogonium und Dimerella (Gyalectaceae). Botanische Jahrbücher für Systematik (no prelo).

Lücking, R. \& Vezda, A. 1998. Taxonomic studies in foliicolous species of the genus Porina (lichenized Ascomycotina: Trichotheliaceae). II. The Porina epiphylla group. Willdenowia 28: 181-225.

Lücking, R.; Sérusiaux, E.; Maia, L. C. \& Pereira, E. C. G. 1998. A revision of names of foliicolous, lichenized fungi published by Batista and coworkers between 1960 and 1975. Lichenologist 30: 121-191.
Lücking, R.; Farkas, E.; Sérusiaux, E. \& Sipman, H. J. M. 1999. Checklist of foliicolous lichens and their lichenicolous fungi. Part I. Foliicolous lichens (extended version). URL: http://www.unibayreuth.de/departments/planta2/research/lichens/homepage.htm (versão V. 1999).

Matzer, M. 1996. Lichenicolous ascomycetes with fissitunicate asci on foliicolous lichens. Mycological Papers 171: IX, I-202.

Santesson, R. 1952. Foliicolous lichens I. A revision of the taxonomy of the obligately foliicolous, lichenized fungi. Symbolae Botanicae Upsalienses 12(1): 1-590.

Sérusiaux, E. 1986. The nature and origin of campylidia in lichenized fungi. Lichenologist 18: 1-35.

Sérusiaux, E. 1989. Foliicolous lichens: ecological and chorological data. Botanical Journal of the Linnean Society 100: 87-96.

Silva, M. da \& Minter, D. W. 1995. Fungi from Brazil. Mycological Papers 169: 1-585.

Singer, R. 1969. Augusto Chaves Batista (1916-1967). Sydowia 22: 343-359.

Vezda, A. 1986. Neue Gattungen der Familie Lecideaceae s. lat. Folia Geobotanica et Phytotaxonomica 21: 199 219.

Whitmore, T. C. 1990. An introduction to tropical rain forests. Clarendon Press, Oxford.

Xavier Filho, L. 1974. Phragmopelthecaceae, uma nova família de microascoliquens. Universidade Federal Rural de Pernambuco, Recife. 